\title{
Ordering of ferromagnetic nanoparticles in nematic liquid crystals
}

\section{DOI:}

10.1039/C7SM01029J

10.1039/C7SM01029J

\section{Document Version}

Accepted author manuscript

Link to publication record in Manchester Research Explorer

\section{Citation for published version (APA):}

Dierking, I., Heberle, M., Osipov, M. A., \& Giesselmann, F. (2017). Ordering of ferromagnetic nanoparticles in nematic liquid crystals. Soft Matter, 13. https://doi.org/10.1039/C7SM01029J, https://doi.org/10.1039/C7SM01029J

\section{Published in:}

Soft Matter

\section{Citing this paper}

Please note that where the full-text provided on Manchester Research Explorer is the Author Accepted Manuscript or Proof version this may differ from the final Published version. If citing, it is advised that you check and use the publisher's definitive version.

\section{General rights}

Copyright and moral rights for the publications made accessible in the Research Explorer are retained by the authors and/or other copyright owners and it is a condition of accessing publications that users recognise and abide by the legal requirements associated with these rights.

\section{Takedown policy}

If you believe that this document breaches copyright please refer to the University of Manchester's Takedown Procedures [http://man.ac.uk/04Y6Bo] or contact uml.scholarlycommunications@manchester.ac.uk providing relevant details, so we can investigate your claim.

\section{OPEN ACCESS}




\section{Accepted Manuscript}

This article can be cited before page numbers have been issued, to do this please use: I. Dierking, M. Heberle, M. Osipov and F. Giesselmann, Soft Matter, 2017, DOI: 10.1039/C7SM01029J.

\section{Soft Matter}

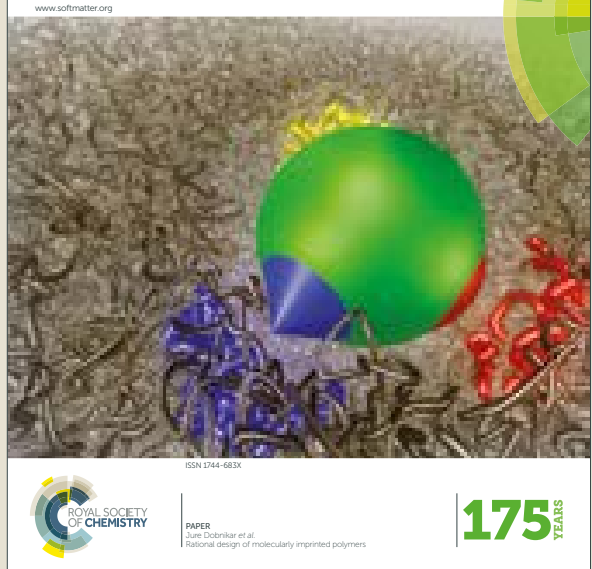

This is an Accepted Manuscript, which has been through the Royal Society of Chemistry peer review process and has been accepted for publication.

Accepted Manuscripts are published online shortly after acceptance, before technical editing, formatting and proof reading. Using this free service, authors can make their results available to the community, in citable form, before we publish the edited article. We will replace this Accepted Manuscript with the edited and formatted Advance Article as soon as it is available.

You can find more information about Accepted Manuscripts in the author guidelines.

Please note that technical editing may introduce minor changes to the text and/or graphics, which may alter content. The journal's standard Terms \& Conditions and the ethical guidelines, outlined in our author and reviewer resource centre, still apply. In no event shall the Royal Society of Chemistry be held responsible for any errors or omissions in this Accepted Manuscript or any consequences arising from the use of any information it contains. 


\title{
Ordering of ferromagnetic nanoparticles in nematic liquid crystals
}

\author{
I. Dierking* ${ }^{1}$, M. Heberle ${ }^{2}$, M.A. Osipov ${ }^{3}$, F, Giesselmann ${ }^{2}$ \\ ${ }^{1}$ School of Physics and Astronomy, University of Manchester, Oxford Road, \\ Manchester M13 9PL, United Kingdom \\ ${ }^{2}$ Institute of Physical Chemistry, University of Stuttgart, Pfaffenwaldring 55, 70569 Stuttgart, \\ Germany \\ ${ }^{3}$ Department of Mathematics, University of Strathclyde, Glasgow G1 1XH, Scotland, United \\ Kingdom
}

* author for correspondence: electronic mail ingo.dierking@manchester.ac.uk 


\begin{abstract}
Dispersions of magnetic nanoparticles in a nematic liquid crystal were investigated as magnetic fields were applied in three different boundary condition geometries: (i) planar substrates and B $\perp \mathbf{n}$, (ii) planar substrates and $\mathbf{B} \| \mathbf{n}$, and (iii) homeotropic substrates and $\mathbf{B} \perp \mathbf{n}$. Particle chaining is observed when a magnetic field is applied, with a periodicity perpendicular to the chains. Furthermore, linear chains are observed for the magnetic field applied perpendicular to the director, while zigzag chains are formed when the magnetic field direction is parallel to the director field. This is attributed to a change from a dipolar defect configuration around dispersed nanoparticles, to a quadrupolar one, i.e. the change from satellite to Saturn-ring defects. This effect is largely independent of the sample thickness. The dynamic development of the chain length, as well as their two-dimensional order parameter was studied in all cases. Chain lengths increased rapidly until saturation at approximately $l=30 \mu \mathrm{m}$ after a time of about $\mathrm{t}=10 \mathrm{~s}$. Similarly, the chain order parameters increased until saturation between $S=0.8-0.9$, independent of sample geometry.
\end{abstract}




\section{Introduction}

In recent years the introduction of nano- and micron-sized particles or clusters into liquid crystal phases has attracted increasing interest from both academic as well as applicational points of view [1]. The main goal is to enhance performance of existing liquid crystal technologies, and to add functionality to be able to develop technologies beyond flat panel displays, spatial light modulators, or optical switches and shutters. Including additional functionality into the selforganized liquid crystal systems opens the huge possibility of novel hybrid materials [2], for example in photonic and sensor applications, but also to study fundamental effects such as pattern formation and induced ordering in anisotropic media [3].

Liquid crystals [4-7] are self-organized anisotropic fluids, thermodynamically located between the low temperature structure of long-range, three dimensional translational and orientational order of a crystal, and the isotropic distribution of the centers of mass of molecules in a liquid with no orientational order (isotropic phase). Liquid crystals exhibit self-organized ordered states, which can range from simple orientational order (nematic) to additional one- or twodimensional translational ordering (fluid and hexatix smectics, respectively). Starting from such a self-ordered system, it is anticipated that this order is transferred onto nano-or micro-inclusions which are shape anisotropic. This has been demonstrated for example in a variety of systems combining nematic [8], smectic [9], lyotropic [10] or discotic [11] liquid crystals with carbon nanotubes. Also other rod-like particles with pronounced aspect ratios have been used, mostly $\mathrm{ZnO}$ nanorods [12,13], but also those prepared from gold[14], or CdSe [15]. The underlying idea in all of these systems is the enhancement of optical, elastic, electric or magnetic responses through shape-anisotropic particles, dispersed and oriented within a liquid crystal matrix. One of 
the main advantages of these systems is further the possibility of a dynamic reorientation of the particles by external stimuli [16].

For completeness, it may also be mentioned that depending on concentration, also liquid crystalline phases can be observed by suspending nanomaterials, such as nanotubes [17], tobacco mosaic viruses [18] or DNA [19], in a suitable isotropic solvent. And that also plate-shaped particles like clays [20] or graphene oxide [21] can be used to achieve similar effects.

On the other hand, also non-elongated particles of dielectric, paramagnetic, super-paramagnetic, ferroelectric or ferromagnetic nature can be employed and dispersed isotropically within different liquid crystal phases [1,22,23]. These particles cannot exhibit a preferred directionality, but they can interact to form anisotropic clusters, exhibit chaining mediated via the interaction of defects formed in the vicinity of the inclusion [3], or form patterns under the application of external fields, like it was studied in this investigation. Previous investigations on liquid crystal based magnetic systems were directed towards nanoparticles [24,25] and observed optic [24] and magneto-optic effects [26], but also rod-like magnetic particles [27]. More recently, ferromagnetism and ferromagnetic ordering was demonstrated for magnetic platelets [26,28,29].

In this paper we study the response of ferromagnetic nanoparticles dispersed at low concentration in a nematic liquid crystal to a static magnetic field in respect to the dynamics and structures of the pattern formation observed. The latter will be compared for three mutually orthogonal geometries. 


\section{Experimental}

The liquid crystal employed in this study is a standard single component room temperature nematic cyanobiphenyl, commonly known as 5CB, commercially available from Sigma-Aldrich. Its phase sequence is Cryst. 22 N 35 Iso., and it was used as purchased. The magnetic anisotropy of $5 \mathrm{CB}$ is positive, so that the director orients parallel to the direction of an applied magnetic field. It should be pointed out, that the investigations presented below were performed at magnetic field strengths below the magnetic Freedericksz threshold. The ferromagnetic particles were isolated from a common ferrofluid (EFH1 from FerroTec), and are approximately $10 \mathrm{~nm}$ in size, while they do cluster to micrometer size to form aggregates which are visible in optical microscopy. As the commercial ferrofluid from which the magnetic particles are dried exhibits a very good dispersion quality, it is believed that they are surfactant coated, as it is often done to improve dispersibility, nevertheless, no conclusive information was available from the ferrofluid producer.

The sandwich cells used were purchased from AWAT (Poland) with defined planar and homeotropic boundary conditions. They were used at different cell gaps between 5-25 $\mu \mathrm{m}$, as indicated in the discussion below. A permanent magnet with a magnetic flux of $0.1 \mathrm{~T}$, uniform over a large area of $50 \mathrm{~cm}^{2}$, was employed to apply magnetic fields to various cell geometries. These were orthogonal to each other: (i) Planar boundary conditions, magnetic field perpendicular to the director, (ii) planar boundary conditions, magnetic field parallel to the director, and (iii) homeotropic boundary conditions, magnetic field perpendicular to the director (cf. Fig. 1) 
Images were captured by a digital camera (Nikon CoolPix 990) with a resolution of 2048x1536 pixels, connected to a polarizing microscope (Olympus BH-2). Both crossed and parallel polarizer conditions were employed. After calibration, statistical image analysis was performed with software IMAGETOOL3, developed at the University of Texas Health Science Center, San Antonio. Chain lengths and chain angles were determined as a function of time of applied magnetic field, and the time development of $2 \mathrm{D}$ order parameters $\mathrm{S}=<2 \cos ^{2} \beta-1>$ were evaluated from experimentally determined order distribution functions, with $\beta$ being the angle between the main particle chain direction and the director or the long side of the sandwich cell in the case of homeotropic alignment.

\section{Experimental Results and Discussion}

The three employed sample geometries are schematically shown in figure 1, where the directions of the magnetic field $\mathbf{B}_{\mathbf{0}}$ and the director $\mathbf{n}$ are indicated. In case (i) planar boundary conditions are applied, with the director parallel to the substrate and the magnetic field perpendicular to the rubbing direction, thus also perpendicular to the director (geometry A). In (ii) planar boundary conditions are used with the magnetic field along the rubbing direction and the director (geometry B), and in (iii) homeotropic boundary conditions are employed with the director perpendicular to the substrate and the magnetic field perpendicular to the director (geometry C). As a nematic liquid crystal is used, the rubbing direction here also indicates the direction of the director. Figure 2 depicts a texture illustration of a typical experimentally obtained image for geometry A, a sandwich cell filled with a dispersion of liquid crystal and magnetic nanoparticles. The individual nanoparticles are typically of a size of $10 \mathrm{~nm}$, which implies that particle 
aggregates are observed here. After the application of a magnetic field a clear chaining of nanoparticles is observed in direction of the magnetic field, which becomes more easily visible when using parallel polarisers (figure2(b)) instead of crossed ones (figure 2(a)). Furthermore, a certain periodicity of approximately $60 \mu \mathrm{m}$ between the nanoparticle chains is observed, as demonstrated by a horizontal cut through figure 2(b) as indicated, plotting the dependence of light intensity as a function of distance. The reason for this periodicity is not clear yet, nevertheless it does not seem to be related to the cell geometry.

Figure 3 shows the time development of the length of nanoparticle chains for two different cell thicknesses, a relatively thin cell of $9 \mu \mathrm{m}$ (figure 3(a)) and a thick cell of $25 \mu \mathrm{m}$ (figure 3(b)). In both cases a saturation chain length of approximately $30 \mu \mathrm{m}$ is reached after about $\mathrm{t}=20 \mathrm{~s}$, independent of cell gap. As an example The order distribution functions for both cell gaps are depicted in figures 4(a) and (c), for the thin and the thick cell $(d=9 \mu \mathrm{m}$ and $d=25 \mu \mathrm{m})$, respectively. From these, the time development of the order parameter of nanoparticle chains can be determined as the magnetic field is applied. The thin cell exhibits a slightly higher saturation order parameter of $\mathrm{S}=0.9$, which is reached after approximately $\mathrm{t}=10 \mathrm{~s}$. The saturation order parameter of the thick cell is slightly lower with $S=0.8$, reached after a somewhat longer time of about $\mathrm{t}=20 \mathrm{~s}$. From reproducibility and considering the image analysis procedure, we conservatively estimate the error on the order parameter values to approximately $\Delta S= \pm 0.05$. The time development of the two-dimensional order parameter is shown in figures 4(b) and 4(d) for the thin and the thick cell, respectively.

The situation is qualitatively different for geometry B. Application of a magnetic field results in general chaining of the nanoparticles, but these do not form a linear chain, but rather a zigzag arrangement, as depicted in figure 5 for an image between parallel polarisers. The segments of 
individual zigzag chains are roughly of the same length with about $1=30 \mu \mathrm{m}$ (figure $6(\mathrm{a})$ for the $d=9 \mu \mathrm{m}$ and figure $6(\mathrm{~b})$ for the $d=25 \mu \mathrm{m}$ cells), again, not being related to the cell geometry. In the case of the magnetic field being parallel to the director, and chains forming zigzag patterns, the time for chain length saturation is, in contrast to geometry A, much longer for the thin cell of $\mathrm{d}=9 \mu \mathrm{m}$ than the thick cell of $\mathrm{d}=25 \mu \mathrm{m}$. In the case of zigzag chains being formed, one has to distinguish chain segments pointing in positive and negative direction away from the director, which leads to an order distribution function which is bimodal, as depicted in figures 7(a) and 7(b) for the thin and the thick cell, respectively $(\mathrm{d}=9 \mu \mathrm{m}$ and $\mathrm{d}=25 \mu \mathrm{m})$. From this, two order parameters can be estimated, one for each type of chain section, here called $S_{\text {pos }}$ and $S_{\text {neg. }}$. These are shown again for the thin and the thick cell in figures 7(c), (d) and 7(e), (f), respectively. Both are of the order of $\mathrm{S}_{\mathrm{pos}, \mathrm{neg}}=0.8-0.9$, saturated after very short times, and independent of the direction of chain segment (compare figure 7(c) to (e), and figure 7(d) to (f)).

For geometry $\mathrm{C}$ standard linear chains of nanoparticles are observed. These behave similar to those investigated for geometry A, with the length saturating at approximately $1=30 \mu \mathrm{m}$ after about $\mathrm{t}=20 \mathrm{~s}$, as can be seen from figure 8 . The cell gap used for this geometry is $19 \mu \mathrm{m}$, thus between those gaps investigated for geometry A. Again, the dynamic behavior appears to be largely independent of cell gap. This can also be seen from the exemplary order distribution function shown in figure 9(a), and the calculated order parameter, which saturates at about $\mathrm{S}=$ 0.9 after approximately $\mathrm{t}=10-20 \mathrm{~s}($ figure $9(\mathrm{~b}))$.

In general, it is observed that for sample cell geometries $\mathrm{A}$ and $\mathrm{C}$ the formation of linear chains of ferromagnetic nanoparticles is observed under magnetic field application. This is in contrast to geometry B, where zigzag chains were observed. We conclude that the linear chains are formed when the magnetic field is perpendicular to the director, $\mathbf{B} \perp \mathbf{n}$, while zigzag configurations are 
formed when the magnetic field is parallel to the director, $\mathbf{B} \| \mathbf{n}$. A possible interpretation, which is in line with spherical colloidal particles in liquid crystals, is related to different defects surrounding the nanoparticles. It was observed that linear chains were formed for dipolar satellite defects, while quadrupolar Saturn ring defects led to chains in the zigzag configuration [3] (cf. fig.10). Possibly a magnetic field applied perpendicular to the director does not change the defect configuration except for deformations of the director field, preserving the original satellite defect. A magnetic field applied parallel to the chains may on the other hand change the defect from satellite to Saturn ring, i.e. from dipolar to quadrupolar, leading to zigzag chains, as shown in figure 10 for monodisperse, spherical, colloidal particles [3]. This may be related to a different local director orientation in the Saturn ring and in the satellite defect with respect to the magnetic field The reason why in this experiment the zigzag chains are not as perfectly developed as in experiments with colloids, is due to the fact that the here employed nanoparticles are not spherical, neither do they have a monodisperse size distribution. One notes also that the formation of zigzag chains may be determined by the effective quadrupole-quadrupole interaction between Saturn rings. It has been shown, for example, that the quadrupolequadrupole interaction is responsible for the transition into the so-called herringbone phase in 2D anisotropic fluids composed of anisotropic particles [24]. Such a phase is characterized by the anticlinic order of particles which is very similar to the zigzag configuration in a single chain.

\section{Simple theoretical model}

Let us consider a simple theoretical model of a zigzag chain assuming that each aggregate in the chain possess an effective quadrupole moment and as a result the chain is characterized by a 
quadrupole density Q. The quadrupole-quadrupole interaction between individual aggregates is described by the following interaction potential, assuming that the quadrupole tensors are uniaxial and the primary axes of all quadrupoles are parallel to the same plane in the zigzag configuration of the chain [30]:

$V_{Q Q}(1,2)=Q^{2} \delta l^{2} R^{-5} \cos \left(2 \varphi_{1}+2 \varphi_{2}\right)$

where $Q$ is the quadrupole density, $\delta l$ is the length of the aggregate, $R$ is the distance between aggregates and the angles $\varphi_{i}=\omega_{i}-\theta_{i}, \mathrm{i}=1,2$, where $\omega_{i}$ is the angle between the axis of the quadrupole $i$ and the reference direction and $\theta_{i}$ is the angle between the interparticle vector and the same reference direction.

In a linear segment of the zigzag chain every individual quadrupole interacts with all other quadrupoles within the same segment and also with all quadrupoles in the two adjacent linear segments (see Fig. 10). One notes that if the two interacting quadrupoles belong to the same segment, the angles $\omega_{i}=\theta_{i}=0$ if the reference direction is parallel to the segment assuming that the axes of the quadrupoles are also parallel to the segment. In contrast, if the two quadrupoles belong to the adjacent segments which make an angle $\beta$ with each other, $\omega_{1}=0, \omega_{2}=\beta$ and $\theta=\alpha$ where the angle $\alpha$ is shown in Fig. 10. In this case it is: $\cos (2 \varphi 1+2 \varphi 2)=-\cos (2 \alpha)$. As a result the total quadrupole-quadrupole interaction energy per segments can be written in the form:

$V=V_{a}+2 V_{b}$

Where $V_{a}$ is the interaction between the quadrupoles which belong to the same linear segment: 
$V_{a}=Q^{2} \int_{\delta l}^{l} \int_{\delta l}^{l}\left(h_{1}-h_{2}\right)^{-5} d h_{1} d\left(h_{1}-h_{2}\right) \sim \frac{1}{4} Q^{2} l(\delta l)^{-4}$

Here we have taken into account that $\delta l<<l$, where $h_{l}$ is the coordinate of the quadrupole 1 along the linear segment.

The potential $V_{b}$ is the total interaction between the quadrupoles in the two adjacent segments. It can be estimated by making the simple assumption that the total quadrupoles of the two segments are located at their centers. Then the quadrupole-quadrupole interaction between the two segments, which make an angle $\pi-\beta$ (with $\beta$ shown in fig. 10) can be estimated as

$V_{b} \sim Q^{2} l^{2} \frac{\cos (2 \beta)}{\left.2 l^{2}+2 l^{2} \cos (\beta)\right)^{\frac{5}{2}}}$

It is interesting to note that the potential (3) which describes the quadrupolar interaction within a linear segment is always positive which indicates that the linear chain with sufficiently large quadrupole density should be unstable. In contrast, the quadrupole-quadrupole interaction between adjacent segments, given by eq.(4), can be negative if $\cos (2 \beta)<0$, i.e. if $\beta>\pi / 4$. The minimum of the potential (4) is achieved when $\cos (\beta)=2-\sqrt{6} 6.5$ which corresponds to $\beta \sim 126$ degrees. Thus the angle between the two adjacent segments is approximately 54 degrees which coincides well with the 50-60 degrees obtained from experimental data (fig. 7(a) and (b)) taking into account the simplicity of the model.

So far we have assumed that the length of the linear segment in the zig-zag chain is equal to a length $l$. The value of $l$ can be estimated if we take into consideration that the bend of the chain 
between the two adjacent segments cost an amount of energy which we denote as $\mathrm{E}_{0}>0$. Let us now consider the chain of the total length $L$ which contains $N$ linear segments. Taking into account the quadrupole-quadrupole interaction between different segments, considered above, the difference between the energy of the zigzag chain and the linear chain can in approximation be expressed as:

$\Delta U \sim(N-1) E_{0}+Q^{2} l^{2} \frac{2 \cos (\beta)^{2}-1}{\left(2 l^{2}+2 l^{2} \cos (\beta)\right)^{\frac{5}{2}}}$

where $\cos (\beta)=2-\sqrt{6} 6.5$ and $1=\mathrm{L} / \mathrm{N}$. Minimizing the energy (5) with respect to $N$, one obtains the equilibrium length of the linear segment as

$l \sim Q \sqrt{\frac{3(19-2 \sqrt{6.5})}{E_{0} L^{2}(6-2 \sqrt{6.5})^{\frac{5}{2}}}}$

Experimentally $L \sim 30 \mu \mathrm{m}$ and $l \sim 10 \mathrm{~nm}$, and hence eq.(6) establishes a relationship between the quadrupole density $Q$ and the bent energy $\mathrm{E}_{0}$. However, both parameters are not known at present and thus it is impossible to make further physical conclusions. At the same time eq.(6) indicates that the length of the straight segment $l$ is expected to decrease with the increasing total chain length $L$, i.e. $1 \sim \mathrm{L}^{-3 / 2}$. This relationship can, in principle, be tested experimentally when more data are available. 


\section{CONCLUSIONS}

When subjected to a static magnetic field of small strength, ferromagnetic nanoparticles cluster and form chains in a nematic liquid crystal. The chains are linear in nature for the magnetic field being directed perpendicular to the liquid crystal director. If the magnetic field is directed parallel to the director, zigzag chains are formed. Particle chaining can be explained via interactions between defects formed in the close vicinity of colloidal inclusions in nematics. The fact that linear and zigzag chains are observed in dependence on the applied magnetic field direction with respect to the director field, can be due to a change from a dipolar to a quadrupolar defect configuration, i.e. the change from satellite to Saturn-ring defects. The length of particle chains increases with time until saturation at about $l=30 \mu \mathrm{m}$ after approximately $t=10 \mathrm{~s}$, largely independent of cell gap and cell geometry. The two-dimensional chain orientational order parameter saturates with time of magnetic field application at values of about $S=0.8-0.9$, also practically independent of cell gap and geometry. This is approximately equivalent as could be expected as the $2 \mathrm{D}$ orientational order parameter for the liquid crystal host. 


\section{References}

[1] T. Hegmann, Hao Qi, V.M. Marx, J. Inorg. Organometallic Polym. Mater., 17, (2007), 483

[2] J.P.F. Lagerwall, G. Scalia, Curr. Appl. Phys., 12, (2012), 1387

[3] I. Musevic, M. Skarabot, Soft Matter, 4, (2008), 195

[4] P.J. Collings, M. Hird, Introduction to Liquid Crystals, Taylor\&Francis, London, 1997

[5] S. Chandrasekhar, Liquid Crystals, $2^{\text {nd }}$ ed., Cambridge University Press, Cambridge, 1992

[6] I. Dierking, Textures of Liquid Crystals, Wiley-VCH, Weinheim, 2003

[7] P.G. de Gennes, J. Prost, The Physics of Liquid Crystals, Clarendon Press, Oxford, 1992

[8] I. Dierking, G. Scalia, P. Morales, D. LeClere, Adv. Mater., 16, (2004), 865

[9] K.P. Sigdel, G.S. Iannacchione, Eur. Phys. J. E, 34, (2011), DOI 10.1140/epje/i2011-11034-7

[10] J. Lagerwall, G. Scalia, M. Haluska, U. Dettlaff-Weglikowska, S. Roth, F. Giesselmann, Adv. Mater., 19, (2007), 359

[11] S. Kumar, H.K. Bisoyi, Angew. Chem. Int. Ed., 46, (2007), 1501

[12] J. Branch, R. Thompson, J. W. Taylor, L. Salamanca-Riba, L. J. Martınez-Miranda1, J. Appl. Phys., 115, (2014), 164313

[13] Mu-Zhe Chen, Wei-Sheng Chen, Shie-Chang Jeng, Sheng-Hsiung Yang, YuehFeng Chung, Optics Express, 21, (2013), 29277

[14] S. Umadevi, Xiang Feng, T. Hegmann, Adv. Funct. Mater., 23, (2013), 1393

[15] M.V. Mukhina, V.V. Danilov, A.O. Orlova, M.V. Fedorov, M.V. Artemyev, A.V. Baranov, Nanotechnology, 23, (2012), 325201

[16] I. Dierking, G. Scalia, P. Morales, J. Appl. Phys., 97, (2005), 044309

[17] S. Badaire, C. Zakri, M. Maugey, A. Derre, J.N. Barisci, G. Wallace, P. Poulin, Adv.

Mater. 17, (2005), 1673 
[18] J. Zasadzinski, R.B. Meyer, Phys. Rev. Lett., 56, (1986), 636

[19] F. Livolant, A. Leforestier, Prog. Polym. Sci., 21, (1996), 1115

[20] E. Paineau, K. Antonova, C. Baravian, I. Bihannic, P. Davidson, I. Dozov, M. ImperorClere, P. Levitz, A. Madsen, F. Meneau, L.J. Michot, J. Phys. Chem. B, 113, (2009), 15858

[21] S.H. Aboutalebi, M.M. Gudarzi,_Q.B. Zheng, J.-K.Kim, Adv. Func. Mater., 21, (2011), 2978

[22] Y. Reznikov, O. Buchnev, O. Tereshchenko, V. Reshetnyak, A. Glushchenko, J. West, Appl. Phys. Lett., 82, (2003), 1917

[23] F.H. Li, J. West, A. Glushenko, C. Il Cheon, Y. Reznikov, J. Soc. Inform. Displ., 14 (2006), 523

[24] N. Podoliak, O. Buchnev, O. Buluy, G. D'Alessandro, M. Kaczmarek, Y. Reznikov, T.J. Sluckin, Soft Matter, 7, (2011), 4742

[25] T. Toth-Katona, P. Salamon, N. Eber, N. Tomasovicova, Z. Mitroova, P. Kopcansky, J. Mag. and Mag. Mater., 372, (2014), 117

[26] A, Mertelj, N. Osterman, D. Lisjak, M. Copic, Soft Matter, 10, (2014), 9065

[27] N. Podoliak, O. Buchnev, D.V. Bavykin, A.N. Kulak, M. Kaczmarek, T.J. Sluckin, J. Colloid Interf. Sci., 386, (2012), 158

[28] A. Mertelj, D. Lisjak, M. Drofenik, M. Copic, Nature, 504, (2013), 237

[29] M. Shuai, A. Klittnick, Y. Shen, G.P. Smith, M.R. Tuchband, C. Zhu, R.G. Petschek, A. Mertelj, D. Lisjak, M. Copic, J.E. Maclennan, M.A. Glaser, N.A. Clark, Nature Comm., 7, (2015), 10394

[30] V.M. Kaganer, M.A. Osipov, J. Chem. Phys., 109, (1998), 2600 


\section{Figure captions}

Fig. 1: Schematic illustration of the three different geometries employed. (a) Geometry A with planar boundary conditions and magnetic field perpendicular to the director, $\mathbf{B} \perp \mathbf{n}$, (b) Geometry B, with planar boundary conditions and magnetic field along the director, $\mathbf{B} \| \mathbf{n}$, and (c) Geometry $\mathrm{C}$ with homeotropic boundary conditions and magnetic field perpendicular to the director, $\mathbf{B} \perp \mathbf{n}$. The cell gaps were for geometry A and B: $d=9-25 \mu \mathrm{m}$ and geometry $\mathrm{C}$ : $\mathrm{d}=19 \mu \mathrm{m}$.

Fig. 2: (a) Texture of liquid crystal - magnetic nanoparticle dispersion after field application, with crossed polarizers. Due to numerous Schlieren defects the nanoparticles are not very well visible. (b) The sample between parallel polarizers clearly shows chaining of the magnetic nanoparticles. (c) Perpendicular to the chaining direction a clear periodicity, in this case approximately $60 \mu \mathrm{m}$, is observed.

Fig. 3: Time dependence on magnetic field application of the average length of the nanoparticle chains in Geometry A for cell gap (a) $d=9 \mu \mathrm{m}$ and (b) $d=25 \mu \mathrm{m}$. After approximately $20 \mathrm{~s}$ the chain length reaches saturation at about $1=30 \mu \mathrm{m}$. (The red lines are a guide to the eye).

Fig. 4: (a), (c) Exemplary order distribution function and (b), (d) determined time dependence of the two-dimensional order parameter $S$ for cell gap $d=9 \mu \mathrm{m}((a)$ and (b)) and $d=25 \mu \mathrm{m}((\mathrm{c})$ and (d)). After approximately $10 \mathrm{~s}$ the order parameter reaches a saturation value of about $\mathrm{S}=0.8-0.9$. (The red lines are a guide to the eye). 
Fig. 5: Texture micrograph of a sample of Geometry B between parallel polarizers. With a magnetic field applied parallel to the director, instead of perpendicular to it, the formation of zigzag chains is observed, instead of linear chains. The chains are not perfectly zigzag shaped as for colloidal particles, due to the deviation of the nanoparticles from a spherical shape, and their polydispersion.

Fig. 6: Time dependence on magnetic field application of the average length of the nanoparticle zigzag chain segments in Geometry B for cell gap (a) $d=9 \mu \mathrm{m}$ and (b) $d=25 \mu \mathrm{m}$. After approximately $150 \mathrm{~s}$ for thin cells and $20 \mathrm{~s}$ for thick cells, the chain segment length reaches saturation at about $1=25-35 \mu \mathrm{m}$. (The red lines are a guide to the eye).

Fig. 7: Bimodal distribution function for (a) the $d=9 \mu \mathrm{m}$ and (b) the $d=25 \mu \mathrm{m}$ cell, describing the angular order of the zigzag chain segments in the positive and the negative direction from the director. (c) and (d) are the corresponding two-dimensional order parameters in positive direction from the director, $S_{\text {pos }}$, and (e) and (f) for the negative direction, $S_{\text {neg }}$, for the $d=9 \mu \mathrm{m}$ and $\mathrm{d}=25 \mu \mathrm{m}$ cells respectively. The order parameters quickly reach a saturation value of $\mathrm{S}_{\mathrm{pos}}=\mathrm{S}_{\text {neg }}=0.8-0.9$. (The red lines are a guide to the eye).

Fig. 8: Chain length evolution of the linear chains observed for Geometry C, at a cell gap of $\mathrm{d}=19 \mu \mathrm{m}$ for homeotropic boundary conditions with the magnetic field applied perpendicular to the director. After about $50 \mathrm{~s}$ the chain length reaches saturation at approximately $1=30-35 \mu \mathrm{m}$. (The red line is a guide to the eye). 
Fig. 9: (a) Order distribution function and (b) two-dimensional order parameter $\mathrm{S}$ of ferromagnetic nanosphere chains in a magnetic field for cell Geometry $\mathrm{C}$, at thickness $\mathrm{d}=19 \mu \mathrm{m}$. (The red lines are a guide to the eye).

Fig.10: Director configuration and experimental examples for linear and zigzag chaining of monodisperse, spherical colloidal particles in a liquid crystal, (a) dipolar arrangement and (b) quadrupolar arrangement (after [3], reprinted with permission from the Royal Society of Chemistry). 
(a) geometry A

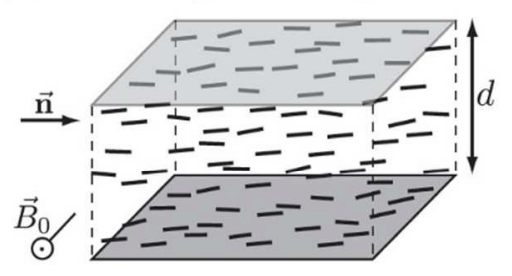

(b) geometry B

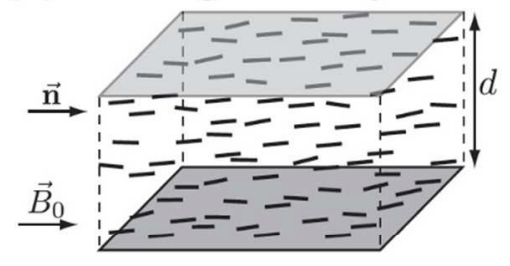

(c) geometry $\mathrm{C}$

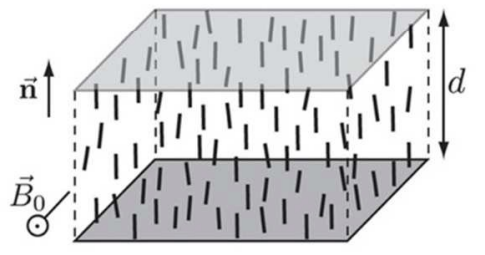

Fig 1 

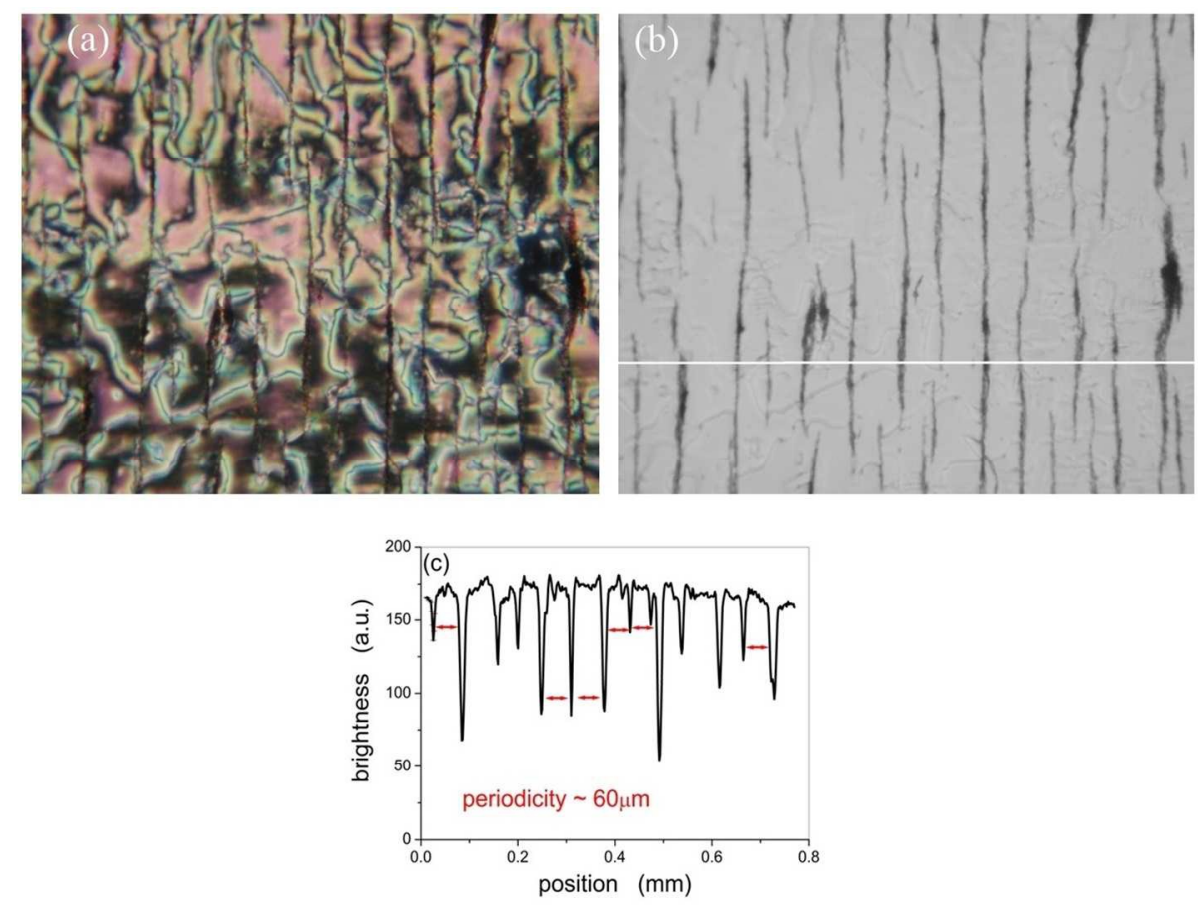

Fig 2 

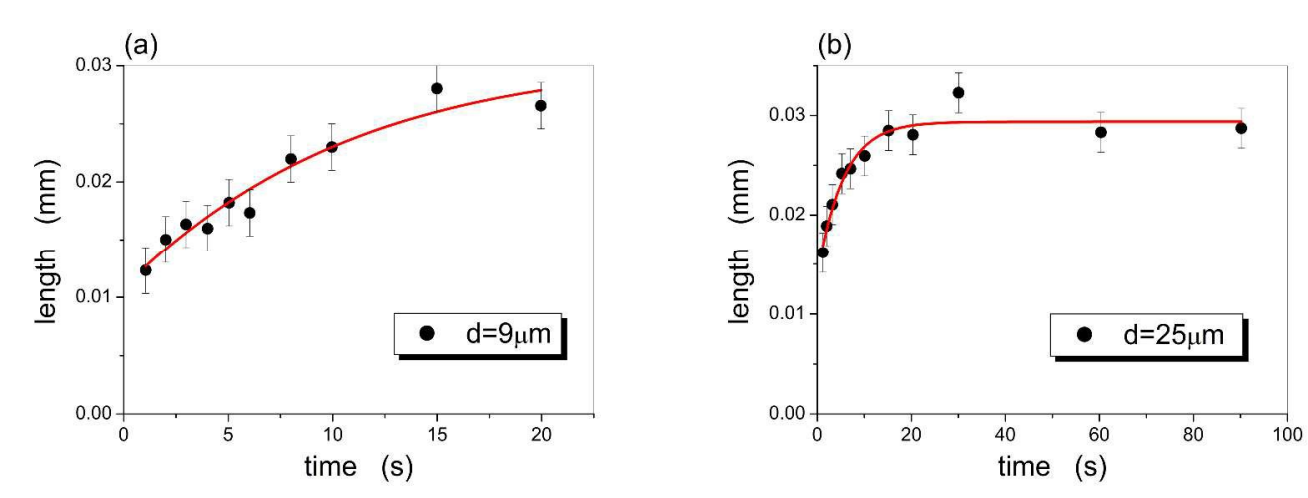

Fig 3 

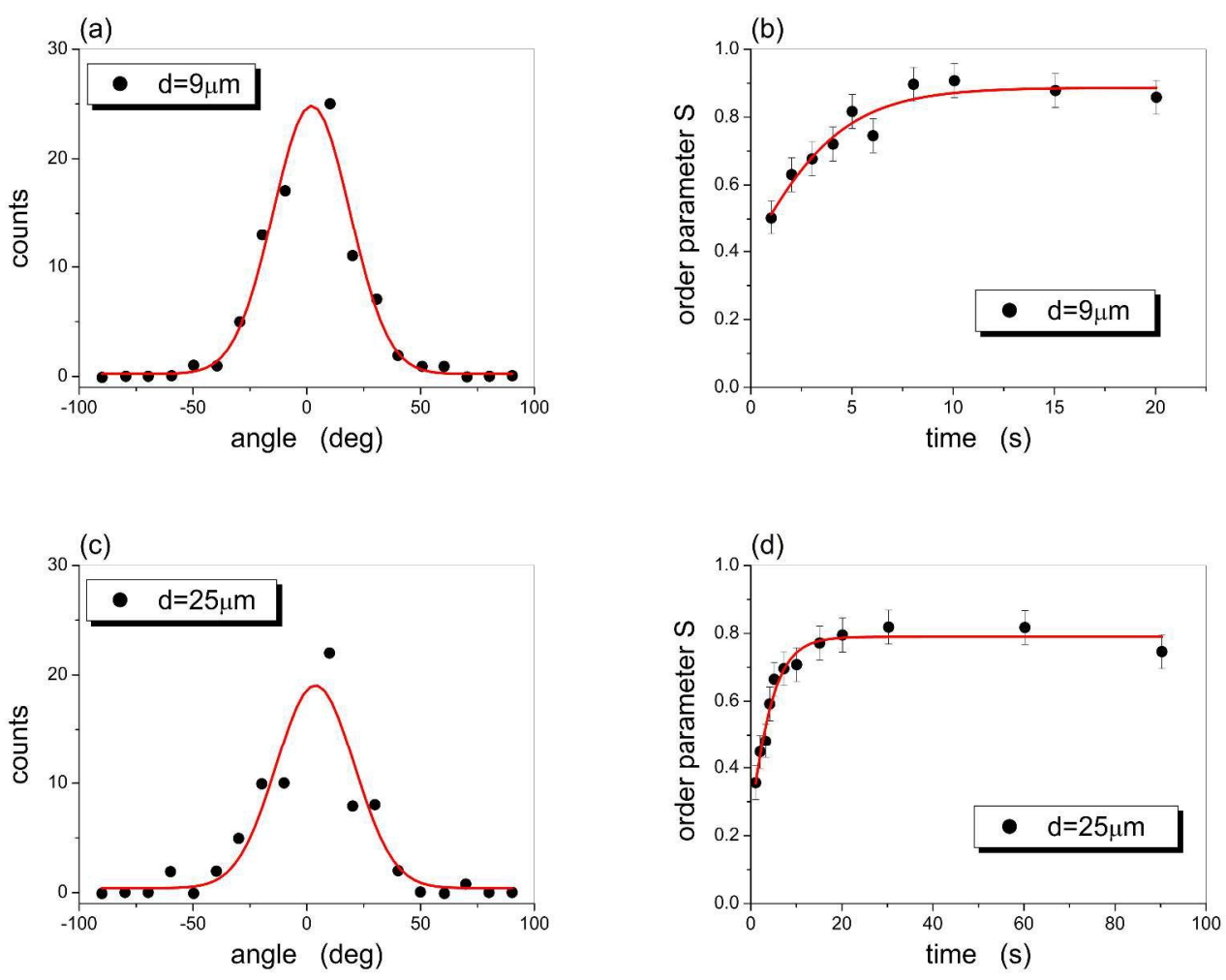

Fig 4 


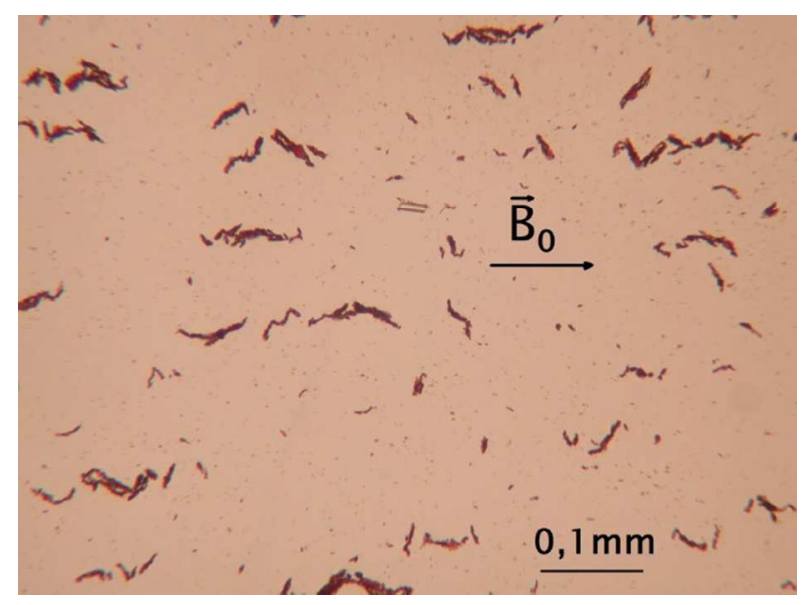

Fig 5 

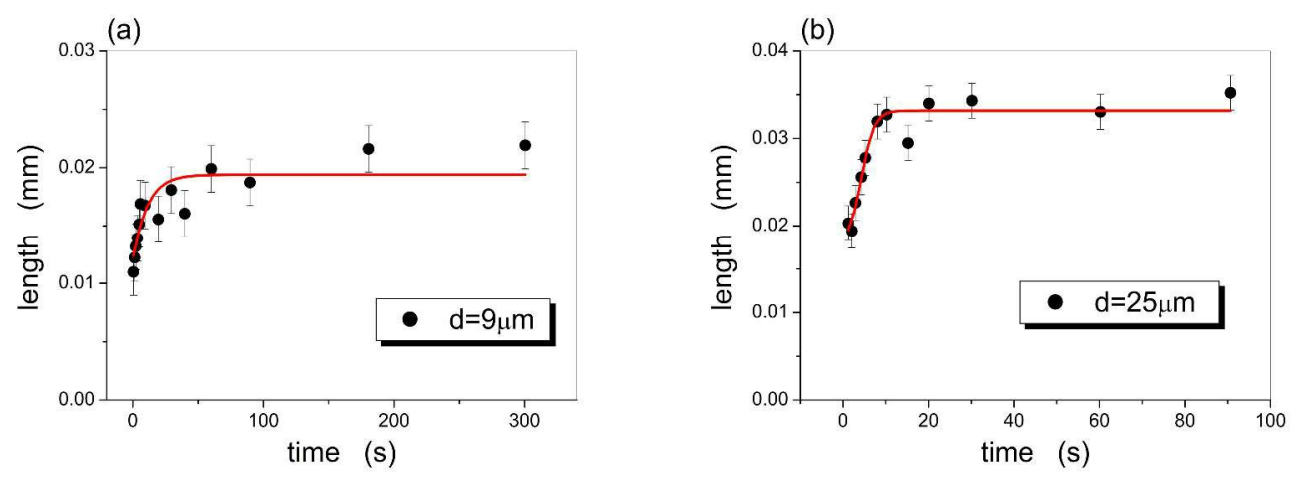

Fig.6 
Page 25 of 29

Soft Matter

View Article Online
DOl: 10.1039/C7SM01029J
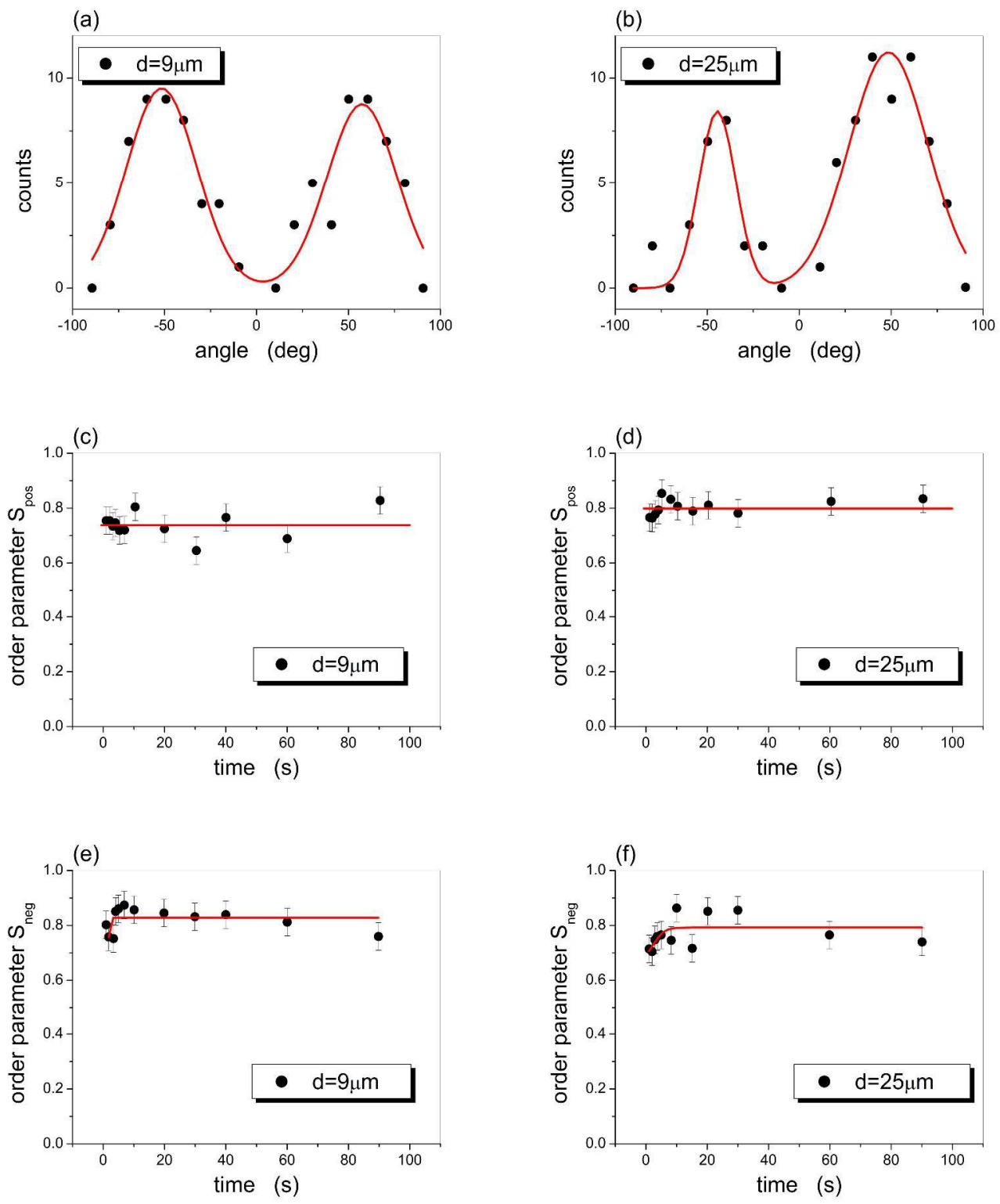

Fig 7 


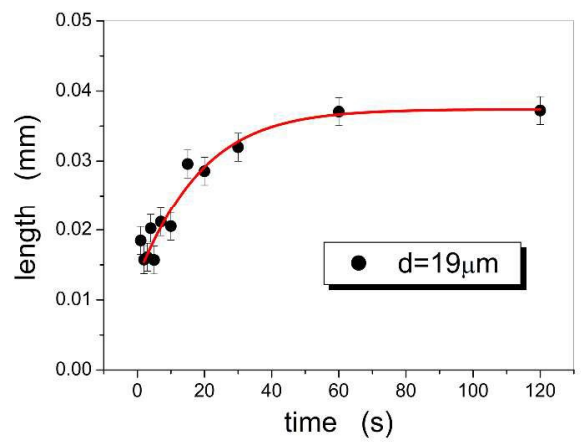

Fig 8 

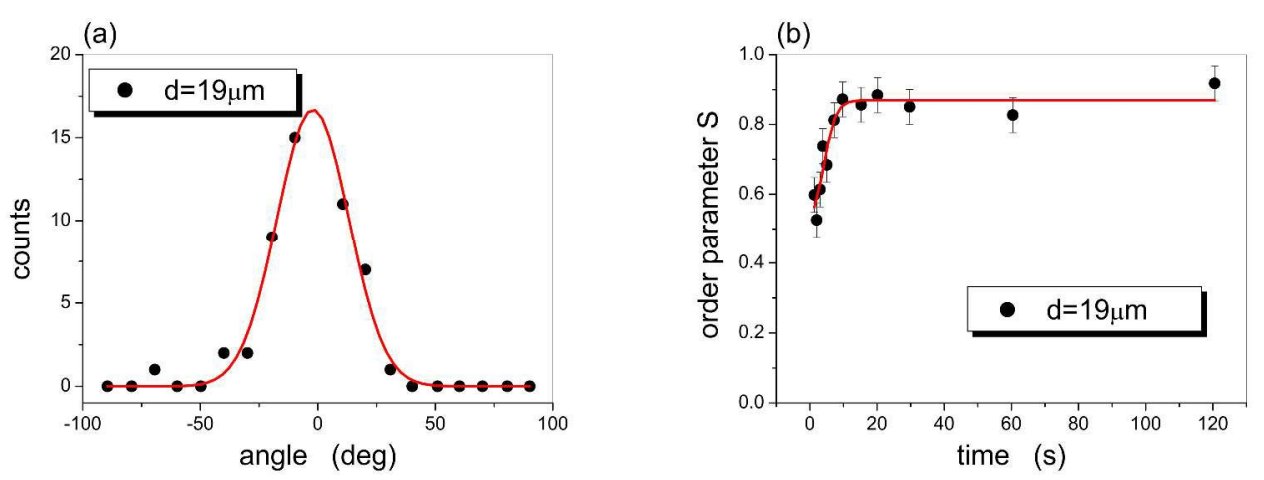

Fig 9 

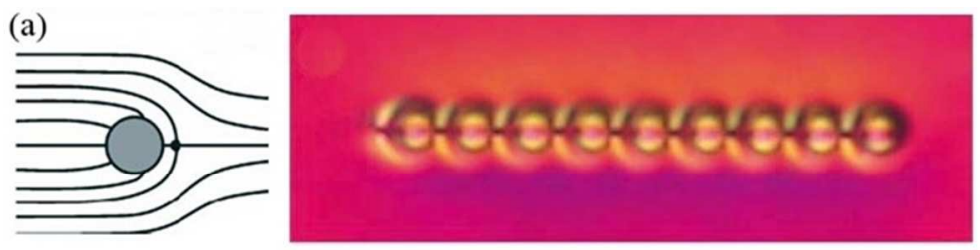

(b)
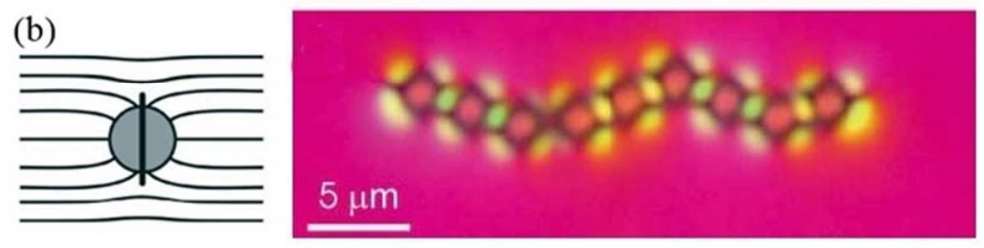

Fig. 10 

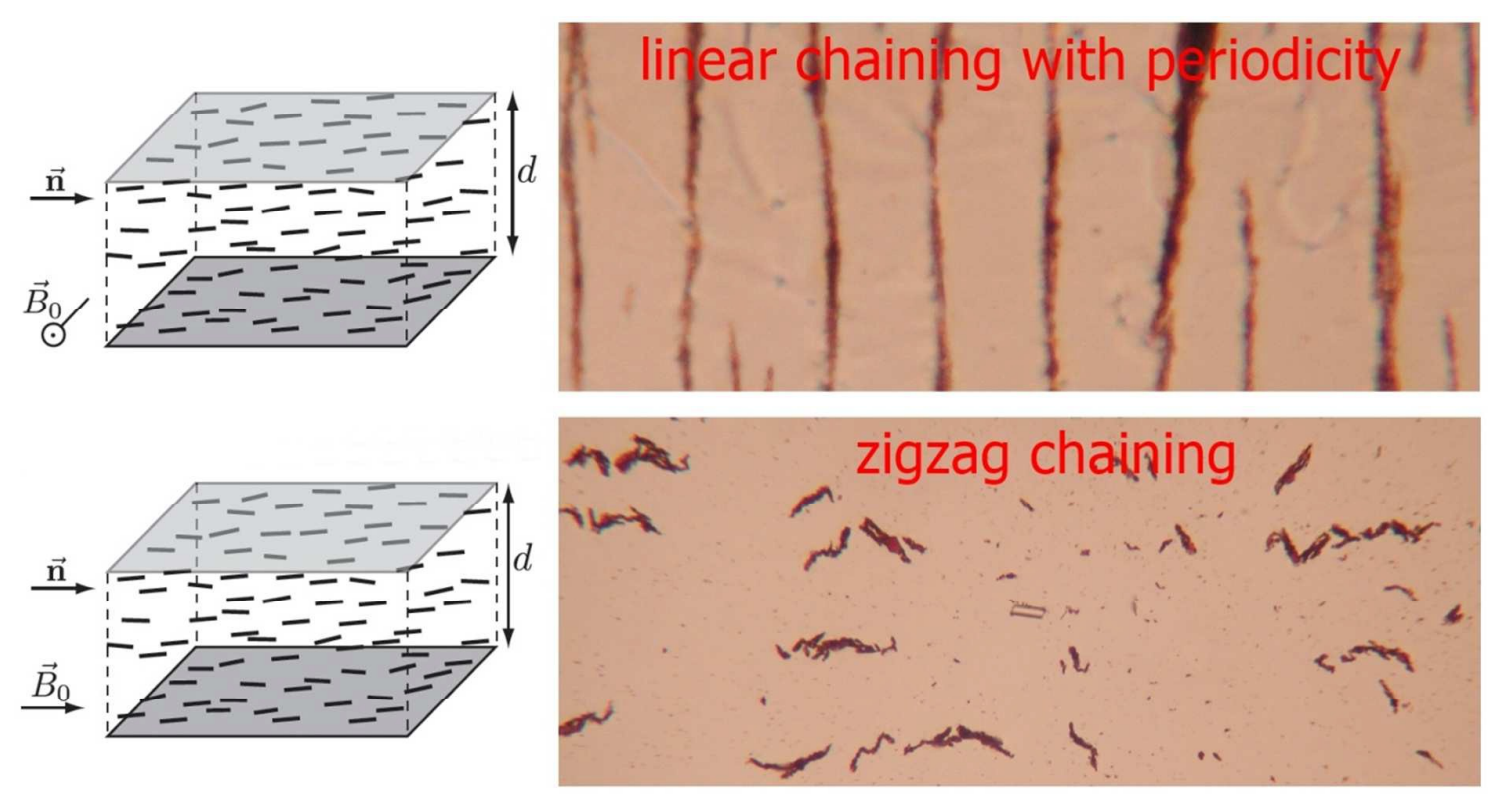

Pattern formation of magnetic particles in a liquid crystal: dynamics, order parameter and periodicity for three perpendicular geometries. 